\title{
Ângulo de Contato e Rugosidade de Madeiras, uma breve revisão
}

\author{
Luis Guilherme Zacharias Sinderski
}

Instituto SENAI de Tecnologia da Madeira e Mobiliário, Arapongas, Paraná, Brasil.

\begin{abstract}
RESUMO Essa revisão discute a molhabilidade de superfícies de madeira através do ângulo de contato, que por sua vez qualifica a molhabilidade e pode fornecer a energia livre, ou tensão da superfície. Discute-se o método da gota séssil por sua simplicidade e relativa precisão para aferir o ângulo de contato. Mesmo que a madeira é caracterizada como um material altamente anisotrópico e heterogêneo é possível verificar o ângulo de contato e assimilar o resultado com outras variáveis da superfície da madeira, como quantidade de extrativos e lignina, a rugosidade superficial e o tipo de lenho da madeira. Tratamentos comuns à madeira também influenciam no ângulo de contato e molhabilidade, pois existe relação evidente entre o lixamento da madeira e a rugosidade assim como os efeitos que que o lixamento causa no ângulo de contato.
\end{abstract}

Palavras-chave: madeira; molhabilidade; ângulo de contato; rugosidade.

\section{Contact angle and roughness of wood, a short review}

\begin{abstract}
This review discusses the wettability of wood surfaces through the contact angle, which in turn qualifies the wettability and can provide free energy, or surface tension. The sessile drop method is discussed for its simplicity and relative accuracy to measure the contact angle. Although the wood is characterized as a highly anisotropic and heterogeneous material, it is possible to verify the contact angle and to assimilate the result with other variables of the wood surface, such as the amount of extractives and lignin, the surface roughness and type of the wood, earlywood or latewood. Treatments common to wood also influence the contact angle and its wettability, since there is a clear relationship between the sanding and the roughness as well as the effects that sanding causes on the contact angle.
\end{abstract}

Keywords: wood; wettability; contact angle; roughness.

\section{Introdução}

Uma das formas de caracterizar a molhabilidade de uma superfície sólida, ou interação entre um líquido e um sólido, se faz através do ângulo de contato entre as duas fases. Quanto menor for o ângulo formado maior é a interação entre as fases, ou maior é a molhabilidade (THAKKER et al., 2013; PRAKASH et al., 2017), assim, ângulos próximos a $0^{\circ}$ indicam que o líquido molha a superfície espontaneamente enquanto que ângulos próximos de $180^{\circ}$ indicam nãomolhabilidade (BARTHLOTT; NEINHUIS, 1997; DE GENNES, 1985). Superfícies altamente hidrofóbicas costumam exibir ângulo de contato na faixa de $160^{\circ}$, racionalizando o "efeito lótus", na qual as gotas de chuva ou de orvalho escorrem rapidamente pela folha mantendo sua superfície sempre seca (CHENG; RODAK, 2005; LIU; CAO, 2018).

A espontaneidade do fenômeno da molhabilidade de superfícies é relacionada com as energias das superfícies envolvidas. Quando a superfície sólida possui energia maior que a energia do liquido, o liquido sofre espalhamento e molha a superfície, ou seja, o ângulo de contato entre o liquido e essa superfície será muito menor que $90^{\circ}$, do contrário, quando a energia da superfície é menor, as 
moléculas do liquido permanecem coalescidas e buscam uma conformação espacial que ofereça maior estabilidade termodinâmica, ou seja, o líquido busca menor contato com a superfície, tomando então o formato próximo de uma esfera e apresentando assim ângulo de contato maior que $90^{\circ}$ (ERBIL, 2014). Interpretação similar pode ser obtida quando se trata das tensões superficiais das substâncias envolvidas no processo de molhabilidade. Embora a interpretação dos fenômenos que envolvem a tensão e a energia das superfícies seja similar, não devem ser tratadas da mesma maneira termodinamicamente, uma vez que a tensão superficial é tratada na interface sólido-líquido-vapor, com unidade dada em N/m, e a energia é dependente da área de contato, sendo sua unidade $\mathrm{em} \mathrm{J} / \mathrm{m}^{2}$ (YUAN; LEE, 2013; DE MEIJER, 2004).

O método da gota séssil é um dos métodos mais comuns e simples de se medir ângulo de contato, na qual uma gota de líquido é depositada em uma superfície lisa e horizontal e o ângulo é medido entre a superfície solida e a tangente do perfil da gota (CHEN; WADA, 1992; ERBIL, 2014), porém existem outros métodos para medir a interação entre as superfícies (YUAN; LEE, 2013).

A medição do ângulo de contato pelo método da gota séssil é feito de forma direta apresentando precisão de $\pm 3^{\circ}$ devido à condições do experimento e da não reprodutibilidade do operador, no entanto ainda é frequentemente preferível do que medições indiretas que podem apresentar erros de diferentes naturezas (STALDER et al., 2006). São utilizados equipamentos específicos, goniômetros equipados com seringas com ponta fina de aço inoxidável ou teflon e um software para se obter o ângulo de contato com maior precisão. Existem outras formas para se realizar essa medição com baixo custo, que utiliza câmera digital, plataforma para o substrato, softwares de fácil acesso (STALDER et al., 2006) e iluminação apropriada, além de uma seringa de precisão, que apresentam resultados aceitáveis e próximos aos equipamentos especializados (THAKKER et al., 2013; STALDER et al., 2006).

Existem outros métodos para medir a interação entre as superfícies. Tal como o método da placa inclinada, na qual uma placa do material em estudo é imersa parcialmente num líquido que forma menisco, a placa é inclinada vagarosamente até o momento em que o menisco fique horizontal e então o ângulo entre a linha horizontal do líquido e a placa é medido. Os métodos que fazem uso do efeito da capilaridade para medir ângulo de contato, como o método do tubo capilar ou da ascensão capilar em placa vertical, fazem a medição do ângulo de contato e da energia da superfície de forma indireta, por meio da mensura da altura que o líquido ascende. Existe também o método da placa de Wilhelmy que mede indiretamente o ângulo de contato numa amostra sólida através da variação da força peso quando a amostra é imersa e retirada do líquido (YUAN; LEE, 2013; GOOD, 1992).

Inicialmente descrito por Young (1804), o ângulo de contato de uma gota de liquido numa superfície lisa é definido pelo equilíbrio mecânico da gota que fica sujeita a ação de diferentes tensões superficiais e pode ser descrita pela Equação 1.

$$
\gamma_{l v} \cos \theta_{Y}=\gamma_{s v}-\gamma_{s l}
$$

onde $\gamma_{l v}, \gamma_{s v}$ e $\gamma_{s l}$ representam as tensões de líquido-vapor, sólido-vapor e sólido-líquido, respectivamente, e $\theta_{Y}$ é o ângulo de contato

A equação (1) é chamada de Equação de Young (DE GENNES, 1985; YUAN; LEE, 2013). No caso do método da gota séssil, é preferível que a gota possua pequeno volume para que a influência da gravidade seja mínima, dessa forma a gota tende a assumir formato esférico (THAKKER et al., 2013; DE GENNES, 1985). Em geral, autores utilizam volumes que variam de 1 a $100 \mu \mathrm{L}$ nesse tipo de ensaio, e 
preferencialmente líquidos puros (THAKKER et al., 2013; CHEN; WADA, 1992; ERBIL, 2014; RODRÍGUEZVALVERDE et al., 2010).

A Equação 1 é válida quando as fases estão em equilíbrio e substratos lisos, mas quando é colocado em questão substratos que possuem defeitos ou quando o liquido é absorvido ou penetra no substrato, são identificadas diferenças entre o ângulo de contato de avanço $\left(\theta_{a}\right)$, logo que o líquido é depositado na superfície, e o ângulo de contato de recuo $\left(\theta_{r}\right)$, quando o liquido por motivo qualquer retrai da superfície recém molhada. $\mathrm{O}$ fenômeno é identificado como histerese de ângulo de contato (DE GENNES, 1985; STALDER et al., 2006; ERBIL, 2014; YUAN; LEE, 2013; RODRÍGUEZ-VALVERDE et al., 2010).

Uma suposta superfície heterogênea de caráter hidrofílico, a grosso modo, pode conter pequenas áreas de caráter hidrofóbico e na medida que a água avança, por exemplo, essas partes retardam o movimento da água aumentando o ângulo de contato, e quando a água recua, essas mesmas partes diminuem ainda mais o ângulo de contato (YUAN; LEE, 2013). É suposto também que a rugosidade do substrato possui influência sobre a molhabilidade, que de acordo com Wenzel (1936) se faz através de um fenômeno termodinâmico, a rugosidade aumenta o espalhamento do líquido com aumento da energia líquida. De maneira geral, quanto maior a energia líquida envolvida, menor é o ângulo de contato, ou seja, menor a interação entre a superfície do substrato e o líquido. No caso da lótus, a planta exibe uma alta rugosidade, porém a causa da hidrofobicidade está relacionada ao desprendimento de partículas da planta que envolvem a gota de água e a impedem de molhar a superfície da folha (BARTHLOTT; NEINHUIS, 1997; CHENG; RODAK, 2005).

Wenzel (1936) foi um dos primeiros a relacionar o ângulo de contato com a rugosidade da superfície através da área na interface líquido-sólido. A teoria proposta sugere que o sólido possui uma área superficial real maior do que a área geométrica por conta da rugosidade desse sólido, portanto a área real da superfície molhada é maior do que a área geométrica, e como já mencionado, essa rugosidade colabora ou não com a molhabilidade através da energia livre apresentada pela superfície em função da diferença topográfica.

Em 1944, Cassie e Baxter estenderam a análise de Wenzel com um novo modelo, na qual descreve um efeito oposto pela presença de regiões não molhadas abaixo de uma gota num sólido de característica hidrofóbica (CASSIE; BAXTER 1944; SINN et al., 2004). Essas regiões não molhadas são geradas a partir de bolhas de ar que ficam presas por entre os sulcos do material rugoso. Esse modelo proposto por Cassie e Baxter se mostrou útil para análise de superfícies heterogêneas, no entanto não é suficiente para explicar a linha de contato na interface tripla (sólido, liquido e gás) corrugada e nem a ampla dispersão dos valores de ângulo de contato observados nos sistemas heterogêneos (ERBIL, 2014). A equação descrita por Cassie e Baxter (Equação 2) leva em consideração a fração de área da superfície $\left(f_{i}\right)$ com o ângulo de contato $\left(\bullet_{i}\right)$ para o ângulo de contato de equilíbrio de um sólido real $\left(\bullet_{e}^{r}\right)$.

$$
\cos \theta_{e}^{r}=\sum f_{i} \cos \theta_{i}
$$

A fração da área de superfície $\left(f_{i}\right)$ contempla a rugosidade da superfície e leva em conta o ar fica preso entre o sólido e o líquido, ou seja, expressa como um fator tanto a área do sólido quanto a área do gás abaixo do líquido que ficam em contato com o último. Em um caso extremo onde a interface líquidogás é zero, então a equação 2 pode ser utilizada para descrever a teoria de Wenzel, uma vez que esta não leva em consideração o ar que fica preso entre o líquido e o sólido por conta da rugosidade (PIAO et al., 2010). Sendo assim, tanto a 
teoria de Wenzel quanto a de Cassie e Baxter procuram estabelecer uma correlação entre o ângulo de contato real, o ângulo de contato real e um fator dependente da rugosidade (SHAKER; SALAHINEJAD 2018).

Por existir certa dificuldade em medir $\theta_{a}$ e $\theta_{r}$, alguns pesquisadores medem apenas o ângulo estático $\left(\theta_{e}\right)$ de uma gota quando essa se encontra livre sobre uma superfície horizontal. O ângulo estático situa-se entre $\theta_{a}$ e $\theta_{r}$, mas não como uma média aritmética e tende ser mais próximo de $\theta_{a}$ do que de $\theta_{r}$ (GOOD, 1992). Em geral, quando existe avanço ou recuo de uma gota é medido o ângulo de contato dinâmico em vez do ângulo estático, sendo que ângulo de contato dinâmico é um termo mais apropriado para materiais que ainda não se encontram em equilíbrio, e suas medidas podem tomadas em função do tempo e tendem a respeitar lei de potência (CHEN; WADA, 1992).

Apesar da nomenclatura, existe uma padronização internacional da American Society for Testing and Materials - ASTM que considera somente dois ângulos de contato na avaliação de recobrimentos, substratos e pigmentos além de outros materiais, sendo o ângulo de avanço e o ângulo de recuo, mas apenas o ângulo de avanço é utilizado na avaliação da padronização com volume de gota menor que $20 \mu \mathrm{L}$ sobre uma amostra orientada horizontalmente. Existe a descrição básica do equipamento necessário para a aferição e as avaliações das superfícies na padronização são embasadas somente no ângulo de contato, dispensando o cálculo da energia superficial livre (ASTM, 2011; ASTM, 2013).

\section{Madeiras e Ângulo de Contato}

A madeira é um material compósito complexo, heterogêneo, rugoso e ainda apresenta poros em sua superfície (GINDL et al., 2001) que torna a medição do ângulo de contato mais complexa do que em superfícies homogêneas e lisas, pois são muitos os fatores que influenciam e ainda estes fatores podem apresentar mensura dificultada.

De forma geral, madeiras apresentam superfícies molháveis pela água $\left(\Theta<90^{\circ}\right)$ a menos que passem por algum tratamento artificial para esse fim (BALKIS et al., 2013; OKON et al., 2017). No entanto, diferentes espécies de madeira podem apresentar diferentes comportamentos em relação ao ângulo de contato por possuírem diferenças nos lenhos primaveris e estivais, na quantidade de lignina e de celulose relata a cada espécie de madeira, uma vez que a lignina tende a ser menos hidrofílica do que a celulose, na quantidade de extrativos e resinas presentes na madeira, na capacidade de absorver água e em sua rugosidade superficial (MANTANIS; YOUNG, 1997).

\section{Extrativos na madeira}

Embora constituída principalmente de celulose (40-50\%), hemicelulose (15-25\%) e lignina (20-35\%), a madeira pode conter ainda $5-15 \%$ de extrativos, que consiste em uma faixa grande de terpenoides, ácidos graxos e substâncias polifenólicas. Tais extrativos, em geral, possuem influência negativa na molhabilidade da madeira (DE MEIJER, 2004). O nome vem da propriedade de serem extraídos da madeira com o solvente apropriado, e desempenham funções metabólicas enquanto que outros são subprodutos e a presença destes no cerne confere resistência à deterioração, no entanto sua oxidação causada pela exposição à luz e ao oxigênio atmosférico leva à mudança de cor na superfície da madeira (BULIAN; GRAYSTONE, 2009).

Existem diversas composições químicas de extrativos, sendo estes classificados em três grandes grupos (BULIAN; GRAYSTONE, 2009; BIERMANN, 2018):

- Compostos fenólicos: encontrados mais comumente no cerne, possuem anéis aromáticos $\mathrm{C}_{6}$ com quantidades variáveis de grupamentos -OH. São os principais 
responsáveis pela coloração escura da madeira e resistência à deterioração.

- Alifáticos: incluem os ácidos graxos e seus ésteres, principalmente os triglicerídeos derivados de ácidos linoleicos, oleicos, linolênicos e esteáricos, com quantidades variando para cada tipo de madeira.

- Terpenos: são uma ampla classe de compostos e normalmente aparecem em grandes quantidades nas madeiras macias, como o pinheiro por exemplo que possui a substância chamada pineno que confere seu cheiro característico. São materiais muito voláteis e oleosos, como o isopreno $\left(\mathrm{C}_{5} \mathrm{H}_{8}\right), \quad$ os monoterpenos $\left(\mathrm{C}_{10} \mathrm{H}_{16}\right)$, os sesquiterpenos $\left(\mathrm{C}_{15} \mathrm{H}_{24}\right)$ e os diterpenos $\left(\mathrm{C}_{20} \mathrm{H}_{32} \mathrm{O}_{2}\right)$, e são utilizados como solventes de tintas e como limpadores domésticos e desinfetantes leves, como a terebintina que é uma mistura de diversos terpenos com maior quantidade de monoterpenos $(\bullet-\mathrm{e} \bullet$-pineno).

Boehme et al. (1996) analisaram 40 diferentes espécies de madeira, tanto europeias quanto tropicais, com o intuito de determinar a dinâmica de uma gota de água destilada nas diferentes superfícies para otimizar processos de colagem, e assumiram que os extrativos presentes na madeira podem aumentar o ângulo de contato e também podem reduzir a absorção de água por parte da madeira.

Papp; Csiha (2017) estudaram quatro espécies de madeiras bastante utilizadas na Hungria, abeto (Picea abies), faia-europeia (Fagus sylvatica L.), bétula-branca (Betula pendula) e carvalho-branco (Quercus petraea) e constataram que o carvalho-branco e o abeto, madeiras com mais extrativos se comparadas a faia-europeia e bétula-branca, apresentaram ângulo de contato maior, sendo portanto, mais hidrofóbicas.

No entanto, a quantidade de extrativos na superfície da madeira é variável e depende do tempo em que a madeira repousa após ser usinada. Nussbaum (1995) e Nussbaum
(1999) estudou esse efeito em abeto (Picea abies) e pinheiroda-Escócia (Pinus silvestres) e chamou de "inativação da madeira", que é quando os extratos do interior da madeira vão para a superfície exposta, tornando-a menos hidrofílica, por consequência aumentando o ângulo de contato com a água. Em seus estudos, Nussbaum utiliza a taxa de molhamento e determina a taxa de ângulo de molhamento constante, e tais taxas tendem a exibir um padrão. Nussbaum também conclui que, para fins de pintura e colagem, madeira de abeto deve ser utilizados até no máximo 2 e 3 dias após o corte, pois exibiram menores tempos de molhabilidade, ou seja, menores ângulos de contatos. $\mathrm{O}$ motivo da movimentação dos extratos do interior da madeira para o exterior, se dá pelo fato de que durante a secagem da madeira, ou mesmo quando esta é cortada, a água volátil que sai durante a secagem arrasta os extrativos que, não volatizados juntos com a água, ficam na superfície (GARDNER, 2016).

A quantidade de extrativos presentes na madeira depende da idade da árvore, indivíduos mais jovens e sem presença do cerne apresentam menos extrativos se comparado à uma árvore da mesma espécie porém com mais idade e com cerne bem formado, uma vez que é nesta região em que se encontram as maiores quantidades de extrativos (DE MELO et al., 2013; ROWELL, 2005).

\section{Lenho Primaveril e Lenho Estival}

Naturalmente existem poros na madeira, mas esses poros possuem diferentes características de acordo com o tipo de lenho. Lenho estival, que cresce durante o verão, tende a ser mais rígido, com maior teor de celulose e pequenos poros. $\mathrm{O}$ lenho primaveril cresce na primavera e apresenta menor densidade, os poros mais são largos e consequentemente esse lenho é mais rugoso (MANTANIS; YOUNG, 1997; DE MEIJER, 2004; PAPP; CSIHA, 2017). A elevada porosidade do lenho primaveril tende a absorver mais o líquido utilizado 
para medição do ângulo de contato do que o lenho estival, e assim o resultado da medição do ângulo de contato no lenho primaveril quando confrontado com uma mesma espécie de madeira na região do lenho estival, ou até mesmo feita a comparação na mesma amostra, será menor, ou seja, o ângulo de contato medido sobre o lenho primaveril é geralmente menor do que o ângulo de contato medido sobre o lenho estival (MANTANIS; YOUNG, 1997; DE MEIJER, 2004), como consequência a energia livre superficial do lenho primaveril é maior.

A fim de evitar contabilizar mais uma variável no estudo, alguns autores realizam as medições do ângulo de contato de forma aleatória ou alternada entre os lenhos, realizando os ensaios em duplicata ou triplicata, ou até mais repetições a fim de reduzir incertezas e estudando o comportamento médio da madeira em relação aos diferentes lenhos (NUSSBAUM, 1995; NUSSBAUM, 1999; PAPP; CSIHA, 2017; SHUPE et al., 2001). Hse (1972) estudou a molhabilidade de resina de fenol-formaldeído em lâminas de pinheiro, discriminando os diferentes tipos de lenho e os resultados demonstraram que o lenho estival molha menos do que o lenho primaveril, portanto, a medição do ângulo de contato de maneira aleatória é válida quando se objetiva estudar processos de pintura e recobrimento uma vez que tanto o lenho primaveril quanto o lenho estival serão recobertos da mesma maneira em tais processos, e depende do tipo de corte das peças de madeira (DE MEIJER 2004; FLEXNER, 2005).

\section{Rugosidade e Superfície}

Como proposto por Wenzel (1936), a rugosidade possui papel importante no espalhamento do líquido que entra em contato com uma superfície, e possui efeito positivo, quanto maior a rugosidade maior o espalhamento de uma gota, no entanto esse efeito é válido até determinada rugosidade, a partir daí o aumento da rugosidade passa a se apresentar de forma negativa e a superfície passa a se apresentar menos hidrofílica. Wenzel utilizou-se do fator de rugosidade " $r$ " para parametrizar a rugosidade da superfície dado pela Equação 3.

$$
r=\frac{\text { superfície atual }}{\text { superfície geométrica }}
$$

Para essa equação, na superfície atual leva-se em conta todos os defeitos e a superfície geométrica é como se apresenta visualmente. Em líquidos a superfície atual e geométrica coincidem e em sólidos a superfície atual é maior que a geométrica. Então a partir da equação de Young, Wenzel propôs uma simples modificação para essa equação que leva em conta o fator de rugosidade (Equação 4).

$$
\gamma_{l v} \cos \theta_{Y}=r\left(\gamma_{s v}-\gamma_{s l}\right)=r A
$$

onde: A é a tensão de adesão.

No entanto, há um extenso debate sobre essa consideração de Wenzel e pouco uso dessa equação (ERBIL, 2014), diversos autores que trabalham com medição de ângulo de contato em madeiras utilizam equipamentos especializados para medir a rugosidade da madeira, como por exemplo rugosímetros e profilômetros (GURAU et al., 2017; LOREDANA; ANNEMARIE, 2015; SLEGERS et al., 2017), ou atacam esse problema de maneira mais simples e fazem o uso de lixas de granulometria conhecida. O fato é que o lixamento é capaz de homogeneizar partes pronunciadas da superfície mas não é capaz de eliminar os vales que a madeira apresenta (HENDARTO et al., 2006).

Nussbaum (1999) verificou que superfícies de abeto e pinheiro-da-Escócia visualmente mais ásperas resultam em maior molhamento e espalhamento da gota séssil de água do que em superfícies planas e lisas. Já Shupe et al. (2001), verificaram que há alguma influência da rugosidade no 
ângulo de contato nas 22 diferentes espécies de madeiras estudadas, no entanto não houve quantificação da rugosidade, apenas o lixamento com lixa de grão 320, e também atribuem que a rugosidade superficial afeta o ângulo de contato na criação de mais de um estado metaestável na interface sólido-líquido-vapor.

Raabe et al. (2017) estudaram lâminas decorativas de curupixá (Micropholis venulosa) com o objetivo de avaliar a rugosidade e a molhabilidade dessas lâminas, foram avaliadas antes e após lixamento e após aplicação de seladora. Embora as lâminas de madeira apresentassem alta heterogeneidade, a rugosidade não se apresentou da mesma maneira nas diferentes amostras em função do processo de maquinação dessas lâminas, que possui forte efeito na rugosidade. Também nesse estudo, foi possível verificar a diminuição dos picos na superfície, mas nenhuma diferença significativa em relação à redução dos vales, no entanto, após a aplicação de seladora formou-se uma superfície altamente homogênea e lisa. Quanto aos ângulos de contato, os autores não obtiveram êxito em medir o ângulo após 60s da aplicação da gota séssil, como estipulado na metodologia que foi utilizada nas amostras sem seladora, pois a água foi completamente absorvida.

A rugosidade da madeira pode ser modificada termicamente, e como consequência a hidrofobicidade, causada pela degradação de componentes da madeira sendo principalmente a degradação da hemicelulose, que reduz a quantidade de grupamentos -OH presentes na superfície que promovem a boa interação entre a água e a madeira. Balkis et al. (2013) estudaram o efeito térmico sobre algumas espécies de madeira e, quando tratadas a $190{ }^{\circ} \mathrm{C}$ por $8 \mathrm{~h}$ apresentaram redução da rugosidade. Quando combinadas a redução da rugosidade com a degradação da hemicelulose, houve incremento da hidrofobicidade de todas as espécies estudadas, com aumento considerável do ângulo de contato da água e as diferentes superfícies.

Outro estudo em que se verifica que uma rugosidade maior em superfícies de madeira ocasiona ângulo de contato menor foi o estudo de De Melo et al. (2013), na qual o objeto de estudo foram lâminas de paricá (Schizolobium amazonicum). Tais lâminas apresentaram diferenças de rugosidades entre seus lados, por conta do processo de maquinação. O lado menos rugoso apresentou maior ângulo de contato se comparado ao lado mais rugoso, avaliado através do método da gota séssil. Porém esse resultado foi mais evidente para a água do que para o adesivo fenolformaldeído. No caso do adesivo, somente os momentos iniciais do ensaio do ângulo de contato apresentaram diferenças quando relacionado à rugosidade, e os autores atribuíram esse comportamento à viscosidade do fluido utilizado, quanto maior a viscosidade menor a penetração do líquido na madeira.

Uma terceira forma de modificar a superfície, e também a rugosidade, da madeira se faz com o uso de plasma frio, nas formas mais comuns de descarga em barreira dielétrica (DBD), corona e jato de plasma a pressão atmosférica (NGUYEN et al., 2018). O plasma frio é uma mistura de partículas excitadas, tais como íons, elétrons e radicais, com baixo grau de ionização e pouca energia de penetração, mas com energia suficiente para romper ligações químicas e deixar a superfície termodinamicamente instável que tende a reagir com os gases presentes na câmara de plasma (ACDA et al., 2012). De acordo com Wolkenhauer et al. (2009) essa técnica permite atingir rugosidade similar ao lixamento, quando comparou nesse trabalho os diferentes tratamentos através da medição da energia superficial de quatro espécies distintas de madeira. Além disso, o tratamento com plasma permite maior controle das características da superfície, não somente a rugosidade, como melhorar a adesão da superfície, aumentar ou diminui a hidrofobicidade e até transformar 
uma superfície em superhidrofóbica (CADEMARTORI et al., 2017).

De fato, Peng; Zhang (2018) fizeram uso de DBD no tratamento de lâminas decorativas de madeira, regulando diferentes parâmetros inerentes ao plasma, tais como velocidade de alimentação de lâmina e intensidade na descarga, concluíram que a rugosidade aumentou após o tratamento, observando as imagens recordadas em microscópio eletrônico de varredura. Com isso, houve um aumento considerável na molhabilidade da superfície, que foi somado à modificação química da superfície pelo aparecimento de grupamentos químicos, em especial grupos polares, no entanto a modificação da química da superfície está diretamente atrelada à espécie da madeira.

Em contrapartida à busca pela melhora da molhabilidade de superfícies de madeira, Cademartori et al. (2017) buscaram aumentar a hidrofobicidade de espécies de madeira através do uso de DBD com descarga de $\mathrm{O}_{2}$ e $\mathrm{C}_{3} \mathrm{~F}_{8}$ e também variando o número de passagens na descarga de plasma. Antes do tratamento com o plasma, as amostras foram lixadas com lixa grã 180, então foi feito a mensura da rugosidade das amostras lixadas e com tratamento de plasma. Comparando os testes de ângulo de contato pelo método da gota séssil, os autores verificaram acréscimo do ângulo após o tratamento com plasma, no entanto, as amostras apresentaram diferenças na rugosidade em função do número de passagens. Apesar do objetivo dos autores de atingir maior hidrofobicidade, um resultado interessante foi obtido, na qual as amostras tratadas mais rugosas apresentaram o menor ângulo de contato, tanto com o uso de $\mathrm{O}_{2}$ quanto com $\mathrm{C}_{3} \mathrm{~F}_{8}$, apesar de serem mais hidrofóbicas do que a amostra não tratada.

Portanto, a rugosidade é um importante parâmetro a ser levado em conta no estudo da madeira, sendo influente no acabamento de pinturas e vernizes, uma vez que a diferença topográfica pode reter bolhas de ar (DE GENNES, 1985) e causar defeitos no recobrimento (FLEXNER, 2005).

\section{Relação entre Lixamento e Ângulo de}

\section{Contato}

A forma de relacionar o lixamento da madeira com o ângulo de contato se faz facilmente através da rugosidade. Como definido por Sandak; Negri (2005), a rugosidade é o resultado da interação entre a madeira de trabalho e a ferramenta, sendo afetada pela geometria e afiação, na qual a anatomia da madeira também possui efeito significante nessa rugosidade. Ou seja, a rugosidade da superfície está diretamente ligada com a ferramenta de trabalho, principalmente com a lixa que é uma ferramenta de acabamento (FLEXNER, 2005).

Relacionando com as ideias de Wenzel (1936), na qual sugere que a rugosidade possui um papel importante no ângulo de contato, é fácil associar essas ideias com o tratamento de lixa na madeira, e de fato o lixamento provoca modificação da energia livre da superfície, logo altera o ângulo de contato. Dependendo da composição da madeira (quantidade de extrativos, lignina, celulose e hemicelulose) as diferentes lixas promovem diferentes efeitos, um lixamento progressivo tende a reduzir o ângulo de contato até um valor mínimo e a partir desse ponto as lixas com grãos mais finos irão aumentar a hidrofobicidade da superfície (PAPP; CSIHA, 2017).

Com o objetivo de recobrir a madeira com tintas ou vernizes é importante ter em mente que um menor ângulo de contato aumenta a energia livre, portanto aumenta a interação entre o líquido e o sólido. Dessa forma, o acabamento deve ser realizado com a lixa que proporciona o menor ângulo de contato, que em geral situa-se entre 120 e 320 dependendo da madeira, e feito de forma progressiva, iniciando com lixa grão 80 ou 100 (FLEXNER, 2005). 
Tratando-se da seringueira (Hevea brasiliansis), somente o grão 120 já é suficiente para proporcionar ângulo de contato ótimo, uma vez que lixada com grãos mais finos o ângulo de contato aumenta (SULAIMAN et al., 2009).

Porém, para um melhor desempenho do recobrimento, além do grau de lixamento deve ser levado em conta a natureza do solvente utilizado. Em geral, os ensaios são realizados com água e a relação entre a morfologia da superfície e o ângulo de contato apresentarão um mínimo em relação à água, portanto, comportamento similar para recobrimentos que possuem água como solvente (DE MELO et al., 2013; KÚDELA, 2014; JAI• et al., 2014).

\section{Considerações Finais}

Embora discutido há muito tempo, o estudo do ângulo de contato em madeiras e seus derivados apresenta muitas variáveis que dificultam o entendimento exato do fenômeno, mas fornece muitas informações pertinentes em relação à superfície da madeira, uma vez conhecida algumas características da superfície em questão, como idade, densidade, espécie, etc. Através do método da gota séssil, a mensura do ângulo de contato se torna simples e rápido, fornecendo informações importantes sobre o comportamento de um ou mais líquidos em relação à espécie de madeira em estudo.

Através do ângulo de contato, é possível a identificação do melhor tratamento a ser utilizado na superfície da madeira para proporcionar maior interação entre o recobrimento e o substrato de madeira, podendo ser uma ferramenta útil e barata em processos industriais, por exemplo, uma vez que a maior molhabilidade de diferentes líquidos sobre a madeira é diretamente correlacionada à maior aderência de filmes de cola ou tinta.

\section{Referências}

ACDA, M. N.; DEVERA, E. E.; CABANGON, R. J.; RAMOS, H. J. Effects of plasma modification on adhesion properties of wood. International Journal of Adhesion and Adhesives, v. 32, n. 1, p. 70-75, 2012.

ASTM INTERNATIONAL PA. ASTM D5727-99, Standard Test Method for Surface Wettability and Absorbency of Sheeted Materials Using an Automated Contact Angle Tester. American Society for Testing and Materials, v. 99, n. Reapproved 2008, p. 1-7, 2011.

ASTM INTERNATIONAL PA. ASTM D7334-08, Standard practice for surface wettability of coatings, substrates and pigments by advancing contact angle measurement: active standard. American Society for Testing and Materials, v. 05.06, p. 1-3, 2013.

BALKIS, B. F.; HIZIROGLU, S.; MD TAHIR, P. Properties of some thermally modified wood species. Materials and Design, v. 43, p. 348-355, 2013. Elsevier Ltd.

BARTHLOTT, W.; NEINHUIS, C. Purity of the sacred lotus, or escape from contamination in biological surfaces. Planta, v. 202, n. 1, p. 1-8, 1997.

BIERMANN, C. J. Wood and Fiber Fundamentals. Biermann's Handbook of Pulp and Paper: Raw Material and Pulp Making. p.19-74, 2018.

BOEHME, B. C.; HORA, G.; WKI, W.; HOLZFORSCHUNG, F. Water Absorption and Contact Angle Measurement of Native European, North American and Tropical Wood Species to Predict Gluing Properties. Holzforschung, v. 50, n. 3, p. 269-276, 1996.

BULIAN, F.; GRAYSTONE, J. A. Wood and Wood-Based Substrates. Wood Coatings: Theory and Practice. p.15-51, 2009.

CADEMARTORI, P. H. G.; STAFFORD, L.; BLANCHET, P.; MAGALHÃES, W. L. E.; DE MUNIZ, G. I. B. RSC Advances Enhancing the water repellency of wood surfaces by atmospheric pressure cold plasma deposition of fluorocarbon film. RSC Advances, p. 29159-29169, 2017.

CASSIE, A. B. D.; BAXTER, S. WETTABILITY OF POROUS SURFACES. Transaction of the Faraday Societ, v. 40, n. 5, p. 546-551, 1944.

CHEN, J.; WADA, N. Edge Profiles and Dynamic Contact Angles of a Spreading Drop. Journal of Colloid and Interface Science, v. 148, n. 1, 1992.

CHENG, Y. T.; RODAK, D. E. Is the lotus leaf superhydrophobic? Applied Physics Letters, v. 86, n. 14, p. $1-3,2005$. 
ERBIL, H. Y. The debate on the dependence of apparent contact angles on drop contact area or three-phase contact line: A review. Surface Science Reports, v. 69, n. 4, p. 325$365,2014$.

FLEXNER, B. Understanding wood finishing. Second ed. Reader's Digest Association, 2005.

GARDNER, D. J. Wood: Surface Properties and Adhesion. Reference Module in Materials Science and Materials Engineering, 2016.

DE GENNES, P. G. Wetting: Statics and dynamics. Reviews of Modern Physics, v. 57, n. 3, p. 827-863, 1985.

GINDL, M.; SINN, G.; GINDL, W.; REITERER, A.; TSCHEGG, S. A comparison of different methods to calculate the surface free energy of wood using contact angle measurements. Colloids and Surfaces A, v. 181, p. 279-287, 2001.

GOOD, R. J. Contact angle, wetting, and adhesion: a critical review. Journal of Adhesion Science and Technology, v. 6, n. 12, p. 1269-1302, 1992.

GURAU, L.; AYRILMIS, N.; THORE, J.; et al. Effect of species and grinding disc distance on the surface roughness parameters of medium density fiberboard. European Journal of Wood and Wood Products, v. 75, n. 3, p. 335-346, 2017.

HENDARTO, B.; SHAYAN, E.; OZARSKA, B.; CARR, R. Analysis of roughness of a sanded wood surface. International Journal of Advanced Manufacturing Technology, v. 28, n. 7-8, p. 775-780, 2006.

HSE, C.-Y. Wettability of Soythern Pine Veneer by Phenol Formaldehyde Wood Adhesives. Forest Products Journal, v. 22, n. I, p. 51-56, 1972.

JAI• , M.; PALIJA, T.; • OR・ EVI•, M. The impact of surface preparation of wood on the adhesion of certain types of coatings. Zaštita materijala, v. 55, n. 1, p. 1-7, 2014.

KÚDELA, J. Wetting of wood surface by a liquids of a different polarity. Wood Research, v. 59, n. 1, p. 11-24, 2014.

LIU, Z.; CAO, J. Fabrication of superhydrophobic wood surface with a silica/silicone oil complex emulsion. Wood Research, v. 63, n. 2, p. 353-364, 2018.

LOREDANA, M. R.; ANNE-MARIE, B. L. Comparative researches on the roughness of sanded wooden surfaces with wide belt and abrasive brushes. Procedia Engineering, v. 100, n. January, p. 1485-1494, 2015.

MANTANIS, G. I.; YOUNG, R. A. Wetting of wood. Wood Science and Technology, v. 31, n. 5, p. 339-353, 1997.

DE MEIJER, M. A review of interfacial aspects in wood coatings: wetting, surface energy, substrate penetration and adhesion. COST E18. Anais... . p.1-16, 2004.

DE MELO, R. R.; SOARES DEL MENEZZI, C. H.; DE SOUZA, M. R.; STANGERLIN, D. M. Avaliação das propriedades físicas, químicas, mecânicas e de superfície de lâminas de paricá (schizolobium amazonicum huber ex. ducke). Floresta e Ambiente, v. 20, n. 2, p. 238-249, 2013.

NGUYEN, T. H. VAN; NGUYEN, T. T.; JI, X.; NGUYEN, V. D.; GUO, M. Enhanced bonding strength of heat-treated wood using a cold atmospheric-pressure nitrogen plasma jet. European Journal of Wood and Wood Products, v. 76, n. 6, p. 1697-1705, 2018.

NUSSBAUM, R. M. The critical time limit to avoid natural inactivation of spruce surfaces (Picea abies) intended for painting or gluing. Holz als Roh- und Werkstoff, v. 53, n. 6, p. $384,1995$.

NUSSBAUM, R. M. Natural surface inactivation of Scots pine and Norway spruce evaluated by contact angle measurements. Holz als Roh - und Werkstoff, v. 57, n. 6, p. 419-424, 1999.

OKON, K. E.; LIN, F.; CHEN, Y.; HUANG, B. Effect of silicone oil heat treatment on the chemical composition, cellulose crystalline structure and contact angle of Chinese parasol wood. Carbohydrate Polymers, v. 164, p. 179-185, 2017.

PAPP, E. A.; CSIHA, C. Contact angle as function of surface roughness of different wood species. Surfaces and Interfaces, v. 8, n. April, p. 54-59, 2017.

PENG, X.; ZHANG, Z. Surface properties of different natural precious decorative veneers by plasma modification. European Journal of Wood and Wood Products, v. 0, n. 0, p. 0, 2018. Springer Berlin Heidelberg.

PIAO, C.; WINANDY, J. E.; SHUPE, T. F. From hydrophilicity to hydrophobicity: A critical review: Part I. Wettability and surface behavior. Wood And Fiber, v. 42, n. 4, p. 490-510, 2010.

PRAKASH, C. G. J.; RAJ, C. C.; PRASANTH, R. Fabrication of zero contact angle ultra-super hydrophilic surfaces. Journal of Colloid And Interface Science, v. 496, p. 300-310, 2017.

RAABE, J.; DEL MENEZZI, C.; GONÇALEZ, J. Avaliação da superfície de lâminas decorativas de curupixá (Micropholis venulosa Mart. Eichler). Floresta e Ambiente, v. 24, n. 180, 2017.

RODRÍGUEZ-VALVERDE, M. A.; RUIZ-CABELLO, F. J. M.; GEA-JÓDAR, P. M.; KAMUSEWITZ, H.; CABRERIZOVÍLCHEZ, M. A. A new model to estimate the Young contact angle from contact angle hysteresis measurements. Colloids 
and Surfaces A: Physicochemical and Engineering Aspects, v. 365 , p. 21-27, 2010.

ROWELL, R. M. Handbook Of Wood Chemistry And Wood Composites. $1^{\circ}$ ed. CRC Press LLC, 2005.

SANDAK, J.; NEGRI, M. Wood surface roughness - what is it? Proceedings of the 17th International Wood Machining Seminar (IWMS 17). Anais... . p.1-10, 2005. Rosenheim, Germany.

SHAKER, M.; SALAHINEJAD, E. A combined criterion of surface free energy and roughness to predict the wettability of non-ideal low-energy surfaces. Progress in Organic Coatings, v. 119, p. 123-126, 2018.

SHUPE, T. F.; HSE, C. Y.; WANG, W. H. An investigation of selected factors that influence hardwood wettability. Holzforschung, v. 55, n. 5, p. 541-548, 2001.

SINN, G.; GINDL, M.; REITERER, A.; STANZL-TSCHEGG, $\mathrm{S}$. Changes in the surface properties of wood due to sanding. Holzforschung, v. 58, n. 3, p. 246-251, 2004.

SLEGERS, S.; LINZAS, M.; DRIJKONINGEN, J.; et al. Surface Roughness Reduction of Additive Manufactured Products by Applying a Functional Coating Using Ultrasonic Spray Coating. Coatings, v. 7, n. 12, p. 208, 2017.

STALDER, A. F.; KULIK, G.; SAGE, D.; BARBIERI, L.; HOFFMANN, P. A snake-based approach to accurate determination of both contact points and contact angles. Colloids and Surfaces A: Physicochemical and Engineering Aspects, v. 286, n. 1-3, p. 92-103, 2006.

SULAIMAN, O.; HASHIM, R.; SUBARI, K.; LIANG, C. K. Effect of sanding on surface roughness of rubberwood. Journal of Materials Processing Technology, v. 209, n. 8, p. 3949-3955, 2009.

THAKKER, M.; JOSHI, R.; SHAH, D. O.; SHUKLA, P. Validation of Low Cost Solid Liquid Contact Angle Instrument Using Drop Shape Image Processing Suitable For Surface Property Measurement. International Journal of Current Engineering and Technology, v. 3, n. 3, p. 877-881, 2013.

WENZEL, R. N. Resistance of solid surfaces to wetting by water. Industrial and Engineering Chemistry, v. 28, n. 8, p. 988-994, 1936.

WOLKENHAUER, A.; AVRAMIDIS, G.; HAUSWALD, E.; MILITZ, H.; VIO, W. Sanding vs . plasma treatment of aged wood: A comparison with respect to surface energy. International Journal of Adhesion \& Adhesives, v. 29, p. 18-22, 2009.

YOUNG, T. An Essay on the Cohesion of Fluids. Philosophical Transactions of the Royal Society of London, v. 95, p. 65-87, 1804.

YUAN, Y. Y.; LEE, R. T. Surface science techniques. Springer Series in Surface Sciences. v. 51, p.3-34, 2013. 